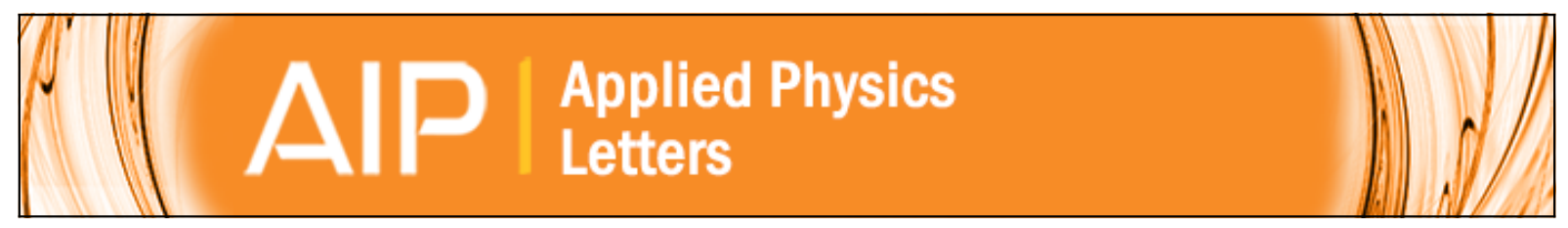

\title{
Magnetization reversal in long chains of submicrometric Co dots
}

J. I. Martn, J. Nogués, Ivan K. Schuller, M. J. Van Bael, K. Temst, C. Van Haesendonck, V. V. Moshchalkov, and

Y. Bruynseraede

Citation: Applied Physics Letters 72, 255 (1998); doi: 10.1063/1.120702

View online: http://dx.doi.org/10.1063/1.120702

View Table of Contents: http://scitation.aip.org/content/aip/journal/apl/72/2?ver=pdfcov

Published by the AIP Publishing

\section{Instruments for advanced science}
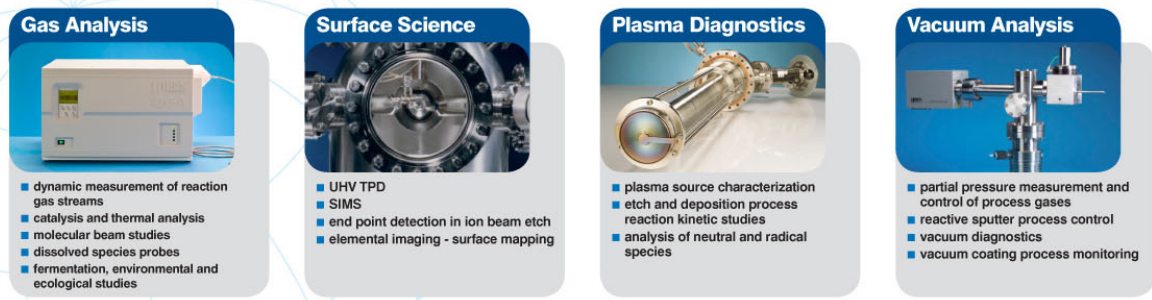

contact Hiden Analytical for further details

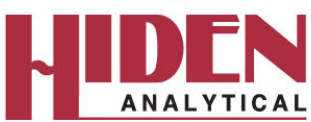

info@hideninc.com www.HidenAnalytical.com CLICK to view our product catalogue 


\title{
Magnetization reversal in long chains of submicrometric Co dots
}

\author{
J. I. Martín, ${ }^{\text {a) }}$ J. Nogués, ${ }^{\text {b) }}$ and Ivan K. Schuller ${ }^{\text {c) }}$ \\ Physics Department, University of California-San Diego, La Jolla, California 92093-0319

\begin{abstract}
M. J. Van Bael, K. Temst, C. Van Haesendonck, V. V. Moshchalkov, and Y. Bruynseraede
Laboratorium voor Vaste-Stoffysica en Magnetisme, Katholieke Universiteit Leuven, Celestijnenlaan 200 D, B 3001 Leuven, Belgium
\end{abstract}

(Received 14 July 1997; accepted for publication 11 November 1997)

\begin{abstract}
Long chains of $400 \mathrm{~nm}$ diam Co dots prepared by combined electron-beam lithography exhibit interesting magnetotransport properties. The magnetoresistance of the chains of dots is markedly different from single Co films, indicating a strongly modified magnetization reversal process. Magnetic force microscopy (MFM) shows that, after magnetic saturation, in the remanent state the single-domain dots are all oriented with their magnetic moment along the chain. A comparison of the magnetoresistance and the MFM reveals that the magnetization reversal occurs by coherent rotation of the magnetic moment in the single-domain dots forming the chain. (C) 1998 American Institute of Physics. [S0003-6951(98)03202-1]
\end{abstract}

The fabrication of submicron magnetic particles with very well-defined geometry is interesting both from the fundamental and the technological point of view. Controlled preparation of submicrometric structures has recently become possible due to the development of techniques like electron-beam lithography, ${ }^{1} \mathrm{x}$-ray lithography, ${ }^{2}$ or scanning probe microscopies. ${ }^{3}$ Moreover, recently developed magnetic force microscopy ${ }^{4,5}$ (MFM) allows analysis of magnetic properties at this small scale. As the particle dimensions become comparable to characteristic lengths such as the magnetic domain-wall size interesting behavior may be expected. In particular, reduced sample dimensions can result in changes of the magnetization reversal processes when compared to bulk material. These processes are very important in applied physics, mainly for their relevance in data storage devices and sensors. ${ }^{6,7}$ The magnetization reversal in arrays of magnetic particles has been studied widely, ${ }^{8-10}$ usually considering a model of "chains of spheres," 11 although this may not be the geometry of the material. Different mechanisms have been proposed such as coherent rotation, ${ }^{12}$ fanning, ${ }^{11}$ curling, ${ }^{13}$ or buckling. ${ }^{13}$ Micromagnetic computations have also predicted a rich variety of magnetization reversal processes in elongated magnetic particles. ${ }^{9}$ It is, therefore, interesting to analyze the behavior in magnetic structures with a well-defined geometry resembling chains of spheres. Recently, chains of iron particles have been synthesized by a chemical method in an aqueous solution, ${ }^{14}$ however, in that case only average properties of the chains could be studied.

In this work, we have studied the magnetization reversal processes in long chains of submicrometric cobalt dots fabricated by electron-beam lithography. In these structures the dots are electrically connected, so that it is possible to carry out transport measurements along the chains. The magnetoresistance has been measured in different magnetic-field configurations, and the results have been compared with

\footnotetext{
a) On leave from Universidad Complutense, 28040 Madrid, Spain.

${ }^{b)}$ On leave from Universitat Autònoma de Barcelona, 08193 Bellatera, Spain.

${ }^{c)}$ Electronic mail: ischuller@ucsd.edu
}

those obtained on reference Co thin films. To develop a better understanding of the magnetization reversal phenomena, the magnetic domains configuration in these chains has been analyzed by MFM.

The samples were fabricated on oxydized Si (100) substrates. A pattern with the desired geometry is defined by electron-beam lithography on a PMMA resist layer. On top of this resist pattern, a Co layer with a thickness of $45 \mathrm{~nm}$ is grown by electron-beam evaporation at room temperature. Under these deposition conditions the Co film is hexagonal and polycrystalline. Finally, the chains of magnetic dots are produced after a lift-off process. The sample consists of 25 parallel chains of dots with a length of $20 \mu \mathrm{m}$ at a distance of $2 \mu \mathrm{m}$ from each other. Each chain contains 50 Co dots. Figure 1 is a scanning electron microscopy (SEM) picture of one of the samples, showing well-defined long chains of Co dots. The dot diameter is approximately $400 \mathrm{~nm}$, which is the same as the distance between the dot centers, while the connections between the dots are about $100 \mathrm{~nm}$ wide. At both ends of the Co chains, rectangular regions have been defined to make the contacts needed for the transport measurements, so that the current flows in parallel through the 25 chains.

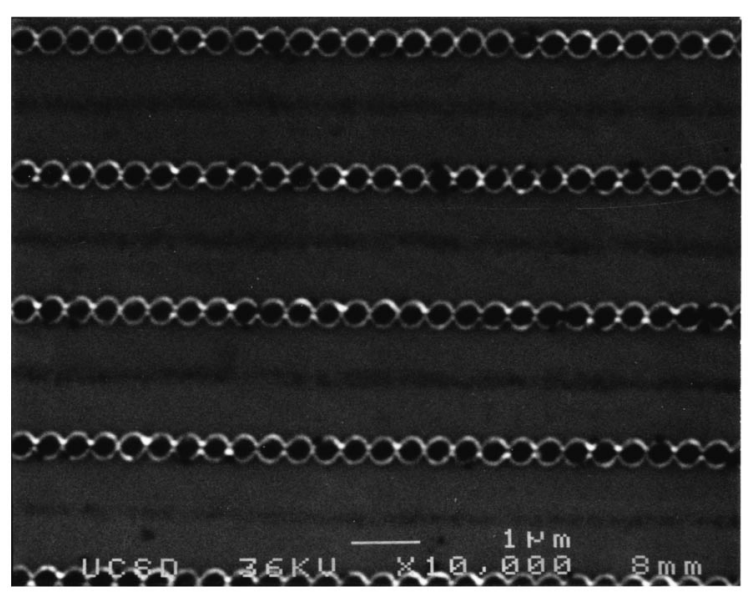

FIG. 1. SEM image of chains of cobalt dots prepared by electron-beam lithography and lift-off technique. The dot size is about $400 \mathrm{~nm}$ in diameter and the distance between chains is $2 \mu \mathrm{m}$. 

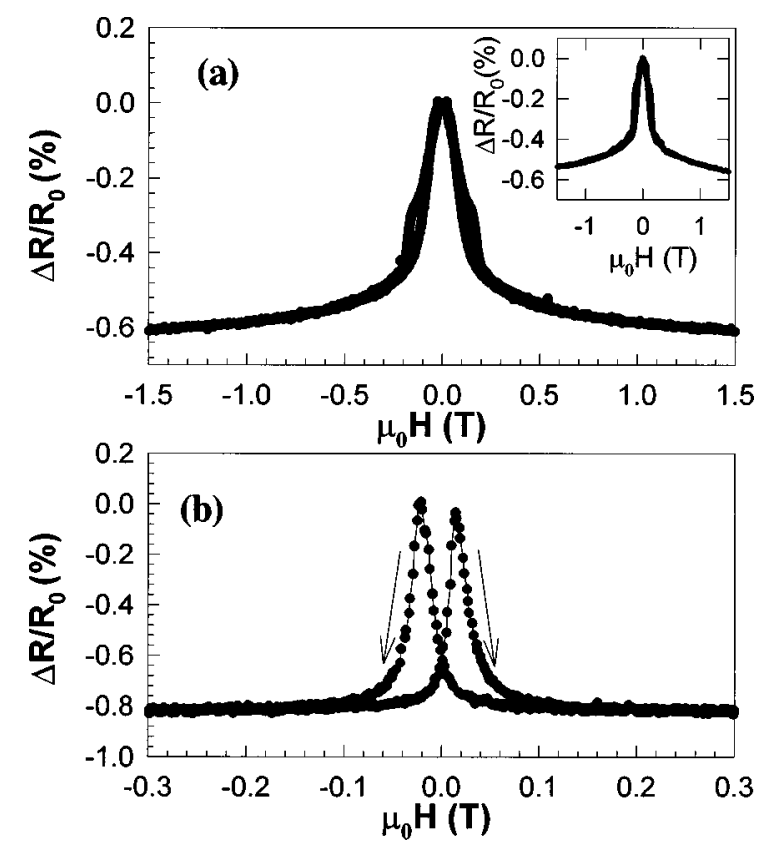

FIG. 2. (a) Resistance ratio vs magnetic field in a sample of chains of Co dots at $T=25 \mathrm{~K}$, with $H$ applied in the substrate plane and perpendicular to the chains. $\left(\Delta R=R-R_{0}\right.$, where $R_{0}$ is the maximum value of the resistance.) The inset corresponds to the same sample measured at $T=250 \mathrm{~K}$. (b) Resistance ratio vs magnetic field in a reference Co film in the same field configuration at $T=25 \mathrm{~K}$.

Contacts are made with silver by conventional optical lithography and an additional lift-off process. Magnetotransport has been measured in a helium cryostat with an $8 \mathrm{~T}$ superconducting magnet and a rotatory sample holder. Several samples have been analyzed showing essentially the same behavior.

A direct high-resolution observation of the magnetic domain structure in the samples has been achieved with MFM. This technique ${ }^{15}$ is similar to atomic force microscopy, but uses a magnetic tip sensitive to magnetic forces. The MFM measurements are carried out on a Nanoscope III from Digital Instruments with commercial magnetic resonant etched silicon nanoprobes. The system is operated in the noncontact mode and the phase detection option. Since the magnetic moment of the tip is directed perpendicular to the scanning plane, the MFM images are dominated by the perpendicular component of the magnetic force gradient between tip and sample. The MFM experiments are performed at room temperature and in zero field. The magnetic domain structure is analyzed in the remanent state before and after saturating the sample magnetization outside the microscope in a $1 \mathrm{~T}$ electromagnet.

For the magnetic field parallel to the substrate plane and perpendicular to the chains of Co dots (i.e., $H$ is perpendicular to the current), the resistance versus field measurements show the characteristic negative magnetoresistance of a ferromagnetic material. ${ }^{16}$ This is illustrated in Fig. 2(a) where the $\Delta R / R_{0}$ versus field data are presented at $T=25 \mathrm{~K}(\Delta R$ $=R-R_{0}$ and $R_{0}$ is the maximum resistance value). As the magnetic field is decreased from the positive saturation, the resistance clearly increases before $H$ switches sign. Actually, it is in the remanent state $(H=0)$ that the resistance approximately reaches its maximum value, and then decreases to saturation for negative fields. In this way this curve is almost identical to the one measured with $H$ going from negative to positive values. This behavior of $\Delta R(H) / R_{0}$ indicates that the magnetization of the cobalt dots starts to rotate when the field is decreased from the saturation, so that their magnetization state in the remanence must have changed considerably when compared to the saturated one. It is important to note that, as presented in the inset of Fig. 2(a), this field dependence of the magnetoresistance observed in the chains of Co dots remains essentially the same when the temperature is increased, at least up to values that are near room temperature (where the MFM measurements are performed). This temperature-independent behavior is not unexpected for a temperature range that is much lower than the Curie temperature of the material. ${ }^{16}$ A comparison with the magnetoresistance of a reference Co thin film of equal thickness grown under the same conditions [see Fig. 2(b)] appears quite different. In the reference Co film, as the magnetic field decreases from saturation, the resistance remains virtually constant, so that it is almost unchanged in the remanent state $(H=0)$; therefore, the magnetization must be similar to the saturated one. Only after $H$ is decreased further reaching negative values, $\Delta R(H) / R_{0}$ increases considerably indicating that the magnetization is rotating. At more negative field, the curve reaches a maximum and decreases towards the negative field saturation state. By varying $H$ from negative to positive values, the complementary behavior is observed, so that the maximum now arises on the positive semiaxis where $\Delta R(H) / R_{0}$ was almost constant during the downward field sweep.

For the measurements carried out with the magnetic field applied parallel to the current (i.e., parallel to the chains of dots), the $\Delta R(H) / R_{0}$ curve has the typical positive magnetoresistance. ${ }^{16}$ However, since in this configuration the field is along the easy direction of magnetization, the resistance value at $H=0$ is still similar to that of the saturation, indicating that the magnetization rotation process takes place mainly after the sign of the field has been reversed.

MFM measurements give further information and confirmation regarding the magnetization rotation processes. Figure 3(a) shows the result of a MFM scan in the remanent state after saturating the magnetization in the direction perpendicular to the chains and in the substrate plane. A periodic magnetic contrast can be clearly observed along the chains of dots, with a periodicity matching the distance between the dot centers. The magnetic image of each dot consists of a white and black spot, which is typical for a singledomain particle with in-plane magnetization, where the white and black regions correspond to the magnetic north and south poles, respectively. Therefore, this result indicates that, in the remanent state, all the Co dots are single domain with the magnetic moment aligned in plane and parallel to the chain direction. It should be noted that the magnetic moment is rotated over $90^{\circ}$ when compared to the imposed saturating field direction. On the other hand, after saturating the sample in a field of $1 \mathrm{~T}$, which is parallel to the chains, the resulting MFM image is similar to the one in Fig. 3(a), indicating that, in this case, the magnetic state of the saturation remains essentially unchanged at the remanence. The observation that the Co dots with this diameter behave as 


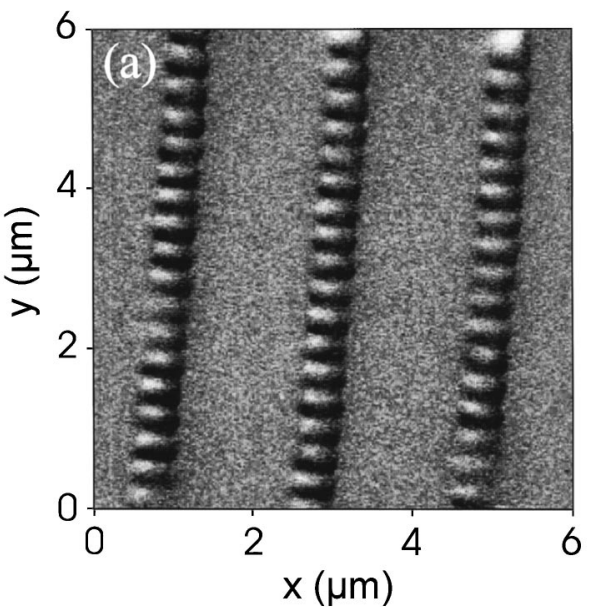

(b)

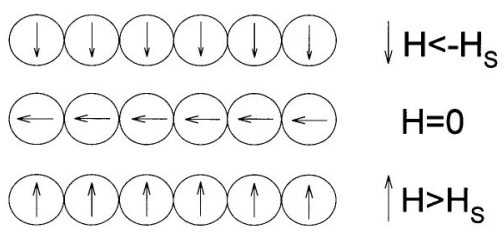

FIG. 3. (a) $6.0 \times 6.0 \mu \mathrm{m}^{2} \mathrm{MFM}$ image of chains of Co dots in the remanent state $(H=0)$ after saturating the sample in a field of $1 \mathrm{~T}$ applied in the substrate plane and perpendicular to the chains direction. (b) Sketch of the coherent rotation mechanism of the magnetization in the chains of magnetic dots when the perpendicular field is swept from $-H_{S}$ to $H_{S}\left(H_{S}\right.$ is the saturation field).

single-domain particles is consistent with the recent MFM results of Gomez et al. ${ }^{17}$ in arrays of isolated dots of similar size. These authors have found different magnetic configurations including single-domain dots. In our geometry, only the single-domain behavior is observed at the remanence.

From the magnetotransport measurements together with the MFM images we can infer that, when the magnetic field is perpendicular to the chains, the magnetization reversal process takes place by coherent rotation, as it is sketched in Fig. 3(b). In this process, each dot behaves as a single domain, which rotates from the negative to the positive saturation state through a configuration at $H=0$ where its magnetic moment is aligned approximately along the chain, which is the easy axis of magnetization. In magnetization reversal processes by coherent rotation, the relation between the saturation magnetization $\left(M_{S}\right)$ and the anisotropy field in the direction perpendicular to the easy axis $\left(H_{K}\right)$ can be written as ${ }^{18} \mu_{0} H_{K}=2 K / M_{S}$, where $K$ is the shape anisotropy constant. Using this equation, we can estimate for the chains of Co dots a value $K \approx 2 \times 10^{5} \mathrm{~J} / \mathrm{m}^{3}$, taking $\mu_{0} H_{K} \approx 0.3 \mathrm{~T}$ from
Fig. 2(a) and $\mu_{0} M_{S}=1.78 \mathrm{~T}$ as the saturation magnetization of cobalt. This high value for $K$ confirms that the shape anisotropy and a coherent rotation process are indeed driving the magnetic behavior in these patterned Co samples.

In summary, we have studied the magnetic properties and the magnetization reversal processes for long and regular chains of Co dots fabricated by electron-beam lithography. The magnetoresistance measurements together with the MFM analysis indicate that, when the magnetic field is applied in the sample plane in the direction perpendicular to the chains, each Co dot behaves as a single magnetic domain and the magnetization is reversed by a coherent rotation process.

This work has been supported by the U.S. Department of Energy, the National Science Foundation, the Fund for Scientific Research-Flanders (FWO), the Belgian InterUniversity Attraction Poles (IUAP), and the Flemish Concerted Action (GOA) research programs. Foreign travel provided by NATO. Two of the authors (J.I.M. and J.N.) acknowledge fellowships from the Spanish Ministerio de Educación y Cultura. One of the authors (M.J.V.B.) is a Research Fellow of the FWO. One of the authors (K.T.) is a Postdoctoral Research Fellow of the FWO. One of the authors (C.V.H.) is a Research Director of the FWO.

${ }^{1}$ J. F. Smyth, S. Schultz, D. R. Fredkin, D. P. Kern, S. A. Rishton, H. Schmid, M. Cali, and T. R. Koehler, J. Appl. Phys. 69, 5262 (1991); A. Maeda, M. Kume, T. Ogura, K. Kuroki, T. Yamada, M. Nishikawa, and Y. Harada, ibid. 76, 6667 (1994).

${ }^{2}$ M. Hehn, K. Ounadjela, J.-P. Bucher, F. Rousseaux, D. Decanini, B. Bartenlian, and C. Chappert, Science 272, 1782 (1996).

${ }^{3}$ D. D. Awschalom, M. A. McCord, and G. Grinstein, Phys. Rev. Lett. 65, 783 (1990).

${ }^{4}$ Y. Martin, and H. K. Wickramasinghe, Appl. Phys. Lett. 50, 1455 (1987).

${ }^{5}$ J. J. Sáenz, N. García, P. Grütter, E. Meyer, H. Heinzelmann, R. Wiesendanger, L. Rosenthaler, H. R. Hidber, and H. J. Güntherodt, J. Appl. Phys. 62, 4293 (1987).

${ }^{6}$ S. Manalis, K. Babcock, J. Massie, V. Elings, and M. Dugas, Appl. Phys. Lett. 66, 2585 (1995).

${ }^{7}$ J. L. Simonds, Phys. Today April 26 (1995).

${ }^{8}$ F. E. Luborsky, J. Appl. Phys. 32, 171S (1961), and references therein.

${ }^{9}$ D. R. Fredkin, T. R. Koehler, J. F. Smyth, and S. Schultz, J. Appl. Phys. 69, 5276 (1991)

${ }^{10}$ M. Lederman, S. Schultz, and M. Ozaki, Phys. Rev. Lett. 73, 1986 (1994).

${ }^{11}$ I. S. Jacobs and C. P. Bean, Phys. Rev. 100, 1060 (1955).

${ }^{12}$ E. C. Stoner and E. P. Wohlfarth, Philos. Trans. R. Soc. London, Ser. A 240, 599 (1948); reprinted in IEEE Trans. Magn. 27, 3475 (1991).

${ }^{13}$ E. H. Frei, S. Shtrikman, and D. Treves, Phys. Rev. 106, 446 (1957).

${ }^{14}$ L. Zhang and A. Manthiram, Phys. Rev. B 54, 3462 (1996).

${ }^{15} \mathrm{R}$. Wiesendanger, Scanning Probe Microscopy and Spectroscopy (Cambridge University Press, Cambridge, 1994).

${ }^{16}$ T. R. McGuire and R. I. Potter, IEEE Trans. Magn. 11, 1018 (1975), and references therein.

${ }^{17}$ R. D. Gomez, M. C. Shih, R. M. H. New, R. F. W. Pease, and R. L. White, J. Appl. Phys. 80, 342 (1996).

${ }^{18}$ B. D. Cullity, Introduction to Magnetic Materials (Addison-Wesley, Reading, MA, 1972). 